\title{
Prescribing issues among older HIV-infected persons in a Mediterranean cohort: Does the current prevalence give cause for concern?
}

\author{
Cora Loste $^{1}$, José Moltó ${ }^{1}$, Núria Pérez ${ }^{1}$, Jordi Puig${ }^{1}$, Patricia Echeverría ${ }^{1}$, Anna Bonjoch ${ }^{1}$, \\ Carmina R.Fumaz ${ }^{1}$, Begoña Lemos ${ }^{1}$, Carla Estany ${ }^{1}$, Bonaventura Clotet $^{1}$, and Eugènia \\ Negredo $^{1}$ \\ ${ }^{1}$ Fundació Lluita Contra la Sida
}

May 11, 2020

\begin{abstract}
AIM: The main objective was to determine the prevalence of prescribing issues in HIV-infected subjects [?]65 years according to the Beers and STOPP/START criteria and drug-drug interactions (Liverpool website). Secondary objectives were to assess the concordance between Beers and STOPP/START criteria in our population, and to identify the drugs most frequently involved in the prescribing issues. METHODS: Cross-sectional cohort study based on a systematic review of the electronic drug prescriptions of $91 \mathrm{HIV}$-infected patients aged [?]65 years. Discrepancies between prescription criteria were assessed using crosstabs and compared using the Chi-square test or Fisher exact test. RESULTS: The mean age was $72.1(5.6)$ years, $75.8 \%$ had [?]3 comorbidities, and 59.3\% polypharmacy. Prescribing issues were identified in $87.9 \% ; 71.4 \%$ by STOPP/START and $45.1 \%$ by Beers. Comparing both criteria, $56.9 \%$ of prescribing issues by STOPP/START were detected by Beers, while $92.5 \%$ of those detected by the Beers criteria were detected by STOPP/START $(\mathrm{p}<0.001)$. Orange/red flag interactions were found in 45.1\%: 3 severe (red) in 2 patients $(2.2 \%)$. The most frequent drugs involved in prescribing issues were benzodiazepines $(>30 \%)$. Cobicistat was the drug most frequently involved in interactions (42.9\%). CONCLUSIONS: The prevalence of prescribing issues among older HIV-infected persons gives cause for concern, as it is almost 90\%. Optimization strategies, including a critical review of the treatment plan, should be implemented in clinical routine by a multidisciplinary team, in particular in patients with multiple comorbidities and polypharmacy. The STOPP/START criteria should be recommended for European populations, since they seem to better detect prescribing issues.
\end{abstract}

\section{INTRODUCTION}

Aging of the general population reveals a series of challenges that must be addressed and resolved. This is especially true with respect to adverse drug reactions (ADRs) in elderly people, including drug-induced morbidity among patients admitted to hospital, which is a major health problem. In addition to the effects on the patient, the consequent increase in health care costs is not inconsiderable. One study estimated total expenditure on medical care related to the use of potentially inappropriate medication to be $\$ 7.2$ billion in the United States in 2001 [1].

Prescribing issues and polypharmacy are risk factors that have been shown to increase the likelihood of ADRs ${ }^{[2.3]}$.

In recent years, several tools have been proposed to help prescribers detect prescribing errors [4-9]. The first explicit criteria were the Beers criteria (1991) [10], which recommended detecting potentially inappropriate medications (PIMs). Despite the widespread use of these criteria throughout the world and the fact that they were updated in 2015, their implementation in Europe has been limited for several reasons: they do 
not consider specific interactions between drugs, therapeutic duplication, the potential prescribing omissions (PPOs) of drugs that are indicated, and the fact that more than $50 \%$ of drugs are not available in Europe. In addition, PIM has not been significantly associated with ADRs in older hospitalized patients according to the Beers criteria [11].

Therefore, in Europe, it was necessary to develop new criteria according to the drugs prescribed in this area. The Screening Tool of Older Persons' Prescriptions (STOPP)/Screening Tool to Alert to Right Treatment (START) [12] criteria were finally published in 2008 by Gallagher et al in Ireland with the objective of detecting potentially inappropriate prescribing (PIP) and PPOs. The European Geriatrics Society contributed significantly to the publication of the criteria in Spain [13] in 2009.

Following the publication of the latest versions of the STOPP-START criteria ${ }^{14}$ and the 2015 revision of the Beers criteria $[5,6,10,15]$, these guidelines are proving to be useful tools, and research on prescribing issues is growing rapidly. In this sense, several studies have shown an association between PIP/PPOs and the risk of ADRs, health-related quality of life, re-hospitalization, and hospital accident and emergency visits in older community-dwelling patients [16-20]. Similarly, there is evidence of a significant improvement in these variables after application of the criteria.

People living with HIV (PLWH) are aging in countries where effective antiretroviral therapy (ART) is available $[21,22]$, and about $50 \%$ of HIV-infected patients are currently more than 50 years old in developed countries. Consequently, patients with multiple comorbidities are becoming more common in daily practice [23]. In fact, the percentage of persons receiving polypharmacy is high in European HIV-infected cohorts (14-40\%) [24-26]. In this context, PLWH could be at risk of prescribing issues. However, despite current knowledge, data on this topic remain scarce, and published data focus mainly on American populations $[27,28]$. Therefore, we performed a study to assess the prevalence of prescribing issues in a Mediterranean cohort of HIV-infected patients aged [?]65 years.

\section{MATERIALS AND METHODS}

\section{Study design and population}

We performed a cross-sectional study based on a systematic review of the most recent electronic prescriptions confirmed by interviewing all patients aged [?]65 years belonging to an HIV-infected cohort (Over50 cohort). The Over50 cohort is an ongoing prospective cohort of chronically HIV-infected people aged [?]50 years consecutively attended in our unit. All patients receive a comprehensive geriatric assessment covering medical, psychological, functional, and social aspects. Written informed consent was obtained from all participants before inclusion. The trial protocol was reviewed and approved by the clinical research ethics committee of the Germans Trias i Pujol Hospital (http://www.ceicgermanstrias.cat/) and met the criteria of the Declaration of Helsinki and the requirements of Good Clinical Practice (REF. CEI: PI-16-068).

\section{Study objectives and endpoints}

The main objective of the study was to determine the prevalence of prescribing issues, including: concomitant medication that had to be stopped (PIMs according to the BEERS criteria or PIP according to the STOPP criteria), concomitant medication that the patient should have been receiving but was not (PPOs according to the STOPP/START criteria), and/or potential and relevant drug interactions between concomitant treatment and ART according to the University of Liverpool website (see definitions below). The secondary objectives included determination of the concordance between the Beers and the STOPP/START criteria in our population, as well as identification of the drugs most frequently involved in the prescribing issues.

\section{Definitions}

A prescribing issue must fulfill at least 1 criterion from the STOPP/START and/or Beers criteria and/or be considered a drug-drug interaction (orange or red flags). 
The study was based on the latest version of the STOPP/START criteria (2015) [14], which includes a list of medication classes divided by sections. Briefly, the STOPP criteria cover potentially inappropriate drugs in patients aged [?]65 years, namely, any drug prescribed without an evidence-based clinical indication, any drug prescribed beyond the recommended duration where treatment duration is well defined, and/or any duplicate drug class prescription. These criteria also classify drugs by family, such as antiplatelet/anticoagulant drugs and central nervous system and psychotropic drugs (see supplementary data of the reference 14). The START criteria cover vaccines and medications that patients should take but are not prescribed. These are classified by systems (e.g., cardiovascular and respiratory systems).

We used the 2015 version of the Beers criteria [15], which was drawn up by an expert panel, includes a list of medications and medication classes that should be avoided or used with caution in adults aged [?]65 years, and is divided into 5 categories.

The Liverpool University website (www.hiv-druginteractions.org) was used to identify drug interactions with ART and concomitant medication; only potential interactions (orange flag) and serious interactions (red flag) were included.

Polypharmacy was defined as 5 or more concomitant drugs excluding ART.

Statistical analysis

Quantitative variables were expressed as mean, SD, and range; qualitative variables were expressed as frequencies and percentages. Discrepancies between prescription criteria were assessed using crosstabs and compared using the Chi-square test or Fisher exact test, p-values of $5 \%$ or lower were considered to be statistically significant. The statistical analysis was performed using SPSS 15.0 (SPSS, Inc., Chicago, Illinois, USA).

\section{RESULTS}

The study population comprised 91 patients aged [?]65 years from the Over50 cohort. The mean (SD) age was 72.1 (5.6) years, and 74 patients were men (81.3\%). Sixty-nine patients (75.8\%) had [?]3 comorbidities, and polypharmacy was recorded in 54 patients $(59.3 \%)$.

The characteristics of the patients are presented in table 1.

\section{Prescribing issues}

Prescribing issues (at least $1 \mathrm{PIM} / \mathrm{PIP}, \mathrm{PPO}$, and/or drug-drug interaction) based on any criteria were identified in 80 patients $(87.9 \%)$.

According to the STOPP/START criteria, we observed the following: 65 patients (71.4\%) had inappropriate prescriptions, with a mean (SD [range]) of 1.27 (1.18 [0.00-6.00]) per subject; 45 patients (49.5\%) were taking at least 1 concomitant medication that had to be stopped (PIP) (0.69 [0.85, 0.00-4.00] per patient); and 39 patients $(42.9 \%)$ should have been receiving 1 concomitant medication that they were not receiving (PPO) (0.58 [0.79, 0.00-4.00] per patient).

According to the Beers criteria, 41 patients (45.1\%) had at least 1 PIM (0.78 [1.10, 0.00-5.00] per patient). The results are shown in figure 1.

When both sets of criteria were compared, $56.9 \%$ of the cases in which a prescription issue was detected by the STOPP/START criteria were also detected by the Beers criteria, while $92.5 \%$ of the cases in which a prescription issue was detected based on the Beers criteria were detected by the STOPP/START criteria $(\mathrm{p}<0.001)$.

A total of 83 potentially relevant drug interactions (orange/red flags) were found between ART and concomitant treatment in 41 patients (45.1\%), with a mean (SD [range]) of 0.93 (1.36 [0.00-6.00]); of these, 3 were severe (red) in 2 patients $(2.2 \%)$.

Drugs and drug families associated with prescribing issues 
The drug families associated with prescribing issues according to the STOPP/START and the Beers criteria are described in detail in tables 2 and 3, respectively.

Briefly, when both sets of criteria were taken into account, benzodiazepines were the family that most frequently had to be discontinued; based on the STOPP criteria, benzodiazepines were followed by opioids and anticholinergic agents; according to the Beers criteria, vasodilators, selective serotonin reuptake inhibitors (SSRIs), and non-cyclooxygenase-selective nonsteroidal anti-inflammatory drugs (NSAIDs) were the categories that had to be stopped.

The most frequent drugs that should have been started (PPO) were the seasonal trivalent influenza and the pneumococcal vaccines, as well as vitamin $\mathrm{D}$ and calcium supplements.

The most frequent drug involved with potentially relevant interactions was cobicistat in 20 patients $(22.0 \%)$, followed by dolutegravir in $9(9.9 \%)$, metformin in $8(8.8 \%)$, and mirenal supplements in $5(5.5 \%)$.

The 3 most severe interactions, which affected 2 patients, were elvitegravir/cobicistat with clopidogrel and darunavir/cobicistat with carbamazepine and fluticasone.

\section{DISCUSSION}

The prevalence of prescribing issues-PIMs/PIP, PPOs, and drug interactions - reached almost $90 \%$ and is a cause for concern among older HIV-infected patients. We identified several differences according to the criteria applied to identify these issues.

Older PLWH are often affected by comorbidities and polypharmacy [24-26] and can therefore be considered an at-risk population for prescribing issues and, consequently, for ADRs. However, to our knowledge, only 3 published studies have assessed this issue in PLWH [27-29]. Our results from the Over50 cohort show that almost all of the patients aged [?]65 years presented at least 1 prescribing issue; half had received at least 1 medication that had to be stopped (PIM by Beers or PIP by STOP). This high rate is similar to that detected in San Francisco in the USA [27,28]. One study detected at least 1 PIM in $52 \%$ of patients in a group of 89 PLWH based on the Beers criteria [27]. A similar prevalence was seen in a second study [28], which included a larger sample (211 patients) and compared both the STOPP/START and the Beers criteria, as did we. Of note, prescribing issues were more prevalent when the Beers criteria were applied than when the STOPP criteria were applied ( $63 \%$ vs $54 \%$, respectively). Since the population studied was American, this result was expected. Data from the Swiss cohort [29] show that the prevalence of PIMs in 122 patients aged > 65 years based only on the Beer criteria was lower than that observed among our patients based on the Beers and the STOPP criteria, around one third of the population compared with a half, respectively. More than half of the medications for which the Beers criteria apply are not available in Spain, and this could be one of the reasons why prescribing issues in Europe are more frequently detected with the STOPP criteria than with the Beers criteria [12]. In this sense, these data indicate that the STOPP/START criteria are more appropriate than the Beers criteria for evaluation of prescribing issues in a European population.

Benzodiazepines were the drugs most frequently involved with PIMs in our and the Swiss cohort [29]. These medications are widely prescribed to the patients we attend, and those with long-term effects should be avoided in elderly patients at risk of falls and in those receiving other drugs that act on the central nervous system. In the American population, however, testosterone was the most frequently involved drug, probably owing to more frequent prescription of this drug among older American PLWH. In contrast, vaccines and vitamin D supplements were the recommended drugs least often prescribed. In line with national guidelines, it is also necessary to recommend vaccines and vitamin D supplements among older PLWH. Therefore, it is very important to carefully and regularly check the concomitant treatment of elderly PLWH at each medical visit, especially in cases involving multiple comorbidities and polypharmacy where different prescribers are involved [29-31]. This routine avoids unnecessary medications, adverse events, and drug-drug interactions.

Finally, our results also illustrate the relevance of choosing the most appropriate antiretroviral drug in elderly PLWH. As expected, antiretroviral combinations including the booster cobicistat were more frequently associated with drug-drug interactions. For that reason, these combinations should be proactively monitored. 
Recent studies support the antiviral efficacy and safety profile of ART combinations without a booster and some dual regimens [32-35] in order to decrease the risk of drug-drug interactions associated with boosters and prescription errors, as well as the risk of ADRs. In fact, despite the lack of formal recommendations on optimal therapy for older PLWH, cohort data demonstrate that the presence of comorbidities and polypharmacy led the physician to prescribe unusual ART combinations, mostly dual regimens [36].

Our study is potentially limited by its small sample size and by the use of the previous version of the Beers criteria (although the modifications of the 2015 revision made little difference to our results) [37] . However, the data resulting from our analysis are solid and relevant and add to the scarce published data. In addition, they highlight the need for changes in our routine practice.

In summary, prescribing issues were very prevalent among our older PLWH, with the most frequent being PIMs/PIP that could lead to avoidable ADRs. This high prevalence requires us to optimize treatment strategies by implementing a multidisciplinary critical review of our treatment plan as part of routine practice $[30,38]$. Concomitant medications should be more carefully and regularly monitored, especially in patients with multiple comorbidities and polypharmacy. Boosted antiretroviral combinations in particular should be used with caution. Although no standardized guidelines are available for PLWH, we consider the STOPP/START criteria to be a useful tool for European populations, since they seem to better detect prescribing issues, as they do in the general population.

\section{CONFLICTS OF INTEREST AND SOURCE OF FUNDING}

\section{This study was conducted as part of our routine work.}

All the authors: none to declare any competing financial or non-financial interests in relation to the work described.

\section{ACKNOWLEDGEMENTS AND AUTHOR CONTRIBUTIONS STATEMENT}

Special acknowledgments for Thomas O'Boyle for the test revision.

C.L, J.M., N.P., J.P, P.E, A.B, C.F., B.L., C.E., B.C. and E.N. visited and compiled patient information.

N.P did the statistical analysis.

C.L., J.M. and E.N. wrote the main manuscript text and prepared figures.

All authors reviewed the manuscript.

FIGURE LEGENDS

Figure 1. Prescribing issues

\section{DATA AVAILABILITY STATEMENT}

The data that supports the findings of this study are available in the supplementary material of this article (Tables).

\section{REFERENCES}

1. O'Connor MN, Gallagher P, O'Mahony D. Inappropriate prescribing: criteria, detection and prevention. Drugs Aging. 2012; 29:437-52.

2. Fu AZ, Jiang JZ, Reeves JH, et al. Potentially inappropriate medication use and healthcare expenditures in the US community-dwelling elderly. Med Care. 2007;45:472-476.

3. Atkin PA, Veitch PC, Veitch EM, Ogle SJ. The epidemiology of serious adverse drug reactions among the elderly. Drugs Aging. 1999;14:141-52.

4. Beers MH. Explicit criteria for determining potentially inappropriate medication use by the elderly. An update. Arch Intern Med. 1997 Jul;157(14):1531-6. 
5. Fick DM, Cooper JW, Wade WE, Waller JL, Maclean JR, Beers MH. Updating the Beers criteria for potentially inappropriate medication use in older adults: results of a US consensus panel of experts. Arch Intern Med. 2003 Dec; 8-22;163(22):2716-24.

6. Rancourt C, Moisan J, Baillargeon L, et al. Potentially inappropriate prescriptions for older patients in long-term care. BMC Geriatr. 2004; 15; 4: 9

7. Holt S, Schmeidl S, Thurmann PA. Potentially inappropriate medications in the elderly: the PRISCUS List. Dtsch Arztebl Int 2010; 107 (31-32): 543-51

8. Rognstad S, Brekke M, Fetveit A, et al. The Norwegian General Practice (NORGEP) criteria for assessing potentially inappropriate prescriptions to elderly patients: a modified Delphi study. Scand J Prim Health Care 2009; 27: 153-9

9. Fick D, Semla T, Beizer J, et al. The American Geriatrics Society 2012 Beers Criteria Update Expert Panel. American Geriatrics Society updated Beers criteria for potentially inappropriate medication use in older adults. JAGS 2012. doi: 10.1111/j.1532-5415.2012. 03923.x [online].

10. Beers MH, Ouslander JG, Rollingher I, et al. Explicit criteria for determining inappropriate medication use in nursing home residents. UCLA Division of Geriatric Medicine. 1991; 151(9):1825-32.

11. Hamilton H, Gallagher P, Ryan C,et al. Potentially Inappropriate Medications Defined by STOPP Criteria and the Risk of Adverse Drug Events in Older Hospitalized Patients. Arch Intern Med. 2011;171(11):1013-1019.

12. Gallagher P, Ryan C, Byrne S, et al. STOPP (Screening Tool of Older Persons' Prescriptions) and START (Screening Tool to Alert Doctors to Right Treatment): consensus validation. Int J Clin Pharmacol Ther 2008; 46 (2): 72-83

13. Delgado Silveira E. Muñoz García M, Montero Errasquin B, et al. Inappropriate prescription in older patients: the STOPP/START criteria. Rev Esp Geriatr Gerontol. 2009; 44(5):273-9

14. O'Mahony D, O'Sullivan D, Byrne S, et al. STOPP/START criteria for potentially inappropriate prescribing in older people: version 2. Age Ageing. 2015; 44(2): 213-218.)

15. By the American Geriatrics Society 2015 Beers Criteria Update Expert Panel. American Geriatrics Society 2015 Updated Beers Criteria for Potentially Inappropriate Medication Use in Older Adults. J Am Geriatr Soc. 2015; 63(11):2227-46.

16. Cahir C, Bennett K, Teljeur C, Fahey T. Potentially inappropriate prescribing and adverse health outcomes in community dwelling older patients. Br J Clin Pharmacol. 2014;77(1):201-10.

17. Motl S, Timpe E, Eichner S. Proposal to improve MedWatch: decentralized, regional surveillance of adverse drug reactions. Am J Health Syst Pharm 2004;61:1840-2

18. Blozik E, Born AM, Stuck AE, et al. Reduction of inappropriate medications among older nursing-home residents: a nurse-led, pre/post-design, intervention study. Drugs Aging. 2010;27:1009-17.

19. Dunn RL, Harrison D, Ripley TL. The beers criteria as an outpatient screening tool for potentially inappropriate medications. Consult Pharm. 2011;26:754-63.

20. Thomas RE, Thomas BC. A systematic review of studies of the STOPP/START 2015 and American Geriatric Society Beers 2015 criteria. Curr Aging Sci. 2019; (2):121-154.

21. Wing E. J. HIV and aging. Int J Infect Dis. 2016; 53, 61-68.

22. Smi M, Brinkman K, Geerlings S, et al. Future challenges for clinical care of an ageing population infected with HIV: a modelling study. Lancet InfecT Dis. 2015; 15, 810-818.

23. Ware D, Palella FJ Jr, Chew KW, et al. Prevalence and trends of polypharmacy among HIVpositive and negative men in the multicenter AIDS cohort study from 2004 to 2016. PLoS One. 2018; 11;13(9):e0203890

24. Guaraldi G, Malagoli A, Calcagno A, et al. The increasing burden and complexity of multi-morbidity and polypharmacy in geriatric HIV patients: a cross sectional study of people aged $65-74$ years and more than 75 years. BMC Geriatr. 2018; 18, 99.

25. Hasse B, Ledergerber B, Furrer H, et al. Morbidity and aging in HIV-infected persons: the Swiss HIV cohort study. Clin Infect Dis. 2011; 53, 1130-1139.

26. Schouten J, Wit FW, Stolte IG, et al. Cross-sectional comparison of the prevalence of age-associated comorbidities and their risk factors between HIV-infected and uninfected individuals: the AGEhIV 
cohort study. Clin Infect Dis. 2014; 59, 1787-1797.

27. Greene M, Steinman MA, McNicholl IR, Valcour VJ. Polypharmacy, drug-drug interactions, and potentially inappropriate medications in older adults with human immunodeficiency virus infection. Am Geriatr Soc. 2014; 62(3):447-53.

28. McNicholl IR, Gandhi M, Hare CB, et al. A Pharmacist- Led program Evaluate and Reduce Polypharmacy and Potentially InappropriatePrescribing in Older HIV-Positive Patients. Pharmacotherapy. 2017; 37(12):1498-1506.

29. Courlet P, Livio F, Guidi M. Swiss HIVCohort Study. Polypharmacy, Drug-Drug Interactions, and Inappropriate Drugs: New Challenges in the Aging Population With HIV. Open Forum Infect Dis. 2019; 21;6(12):ofz531.

30. Blanco JR, Morillo R, Abril V, et al. Deprescribing of non-antiretroviral therapy in HIV-infected patients. Eur J Clin Pharmacol. 2020; 76(3):305-318.

31. Negredo E, Back D, Blanco JR, et al. Aging in HIV-Infected Subjects: A New Scenario and a New View. Biomed Res Int. 2017; 2017:5897298.

32. Scott LJ. Dolutegravir/Lamivudine Single-Tablet Regimen: A Review in HIV-1 Infection. Drugs. 2020; 80(1):61-72.

33. van Wyk J, Ajana F, Bisshop F, et al. Efficacy and Safety of Switching to Dolutegravir/Lamivudine Fixed-Dose Two-Drug Regimen Versus Continuing a Tenofovir Alafenamide-Based Three- or FourDrug Regimen for Maintenance of Virologic Suppression in Adults With HIV-1: Phase 3, Randomized, Non-inferiority TANGO Study. Clin Infect Dis. 2020; pii: ciz1243.

34. Dowers E, Zamora F, Barakat LA, Ogbuagu O. Dolutegravir/rilpivirine for the treatment of HIV1 infection. Drugs. 2018; 2;10:215-224. 2018 Nov;78(16):1741-1750.

35. Aboud M, Orkin C, Podzamczer D, et al. Efficacy and safety of dolutegravir-rilpivirine for maintenance of virological suppression in adults with HIV-1: 100-week data from the randomised, open-label, phase 3 SWORD-1 and SWORD-2 studies. Lancet HIV. 2019; 6(9):e576-e587.

36. Nozza S, Malagoli A, Maia L, et al. GEPPO Study Group. Antiretroviral therapy in geriatric HIV patients: the GEPPO cohort study. J Antimicrob Chemother. 2017; 72(10):2961.

37. By the 2019 American Geriatrics Society Beers Criteria@ B Udate Expert Panel. American Geriatrics Society Beers Criteria Update Expert Panel. American Geriatrics Society 2019 Updated AGS Beers

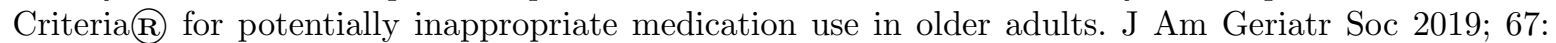
674-94.

38. Livio F, Marzolini C. Prescribing issues in older adults living with HIV: thinking beyond drug-drug interactions with antiretroviral drugs. 2019; 10: 2042098619880122.

\section{Hosted file}

Table 1-LOSTE.docx available at https://authorea.com/users/320453/articles/450001prescribing-issues-among-older-hiv-infected-persons-in-a-mediterranean-cohort-doesthe-current-prevalence-give-cause-for-concern

\section{Hosted file}

Table 2-LOSTE.docx available at https://authorea.com/users/320453/articles/450001prescribing-issues-among-older-hiv-infected-persons-in-a-mediterranean-cohort-doesthe-current-prevalence-give-cause-for-concern

\section{Hosted file}

Table 3-LOSTE.docx available at https://authorea.com/users/320453/articles/450001prescribing-issues-among-older-hiv-infected-persons-in-a-mediterranean-cohort-doesthe-current-prevalence-give-cause-for-concern 


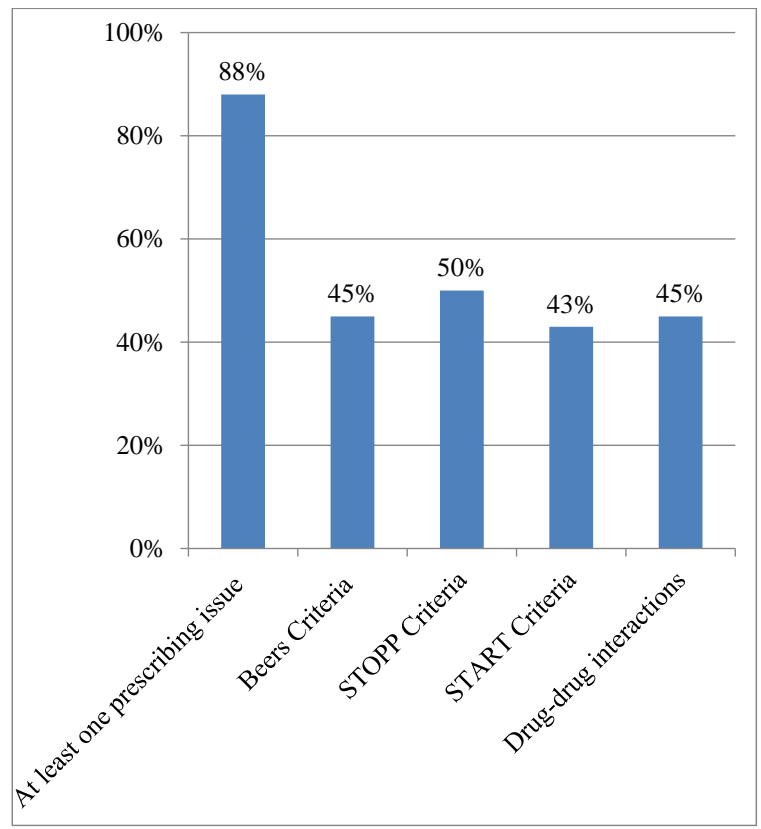

